\title{
The Zen of XEN: insight into differentiation, metabolism and genomic integrity
}

\author{
Mohamed I. Gatie (1),3, Amy R. Assabgui ${ }^{1,2}$ and Gregory M. Kelly $\mathbb{D}^{1,3,4,5,6,7}$
}

\section{From fertilization to implantation}

Fertilization of the mouse egg takes place in the oviduct, and following rounds of cell divisions the blastocyst forms and is comprised of three cell lineages. The epiblast houses naive or preimplantation embryonic stem cells (ESCs), which express OCT4, NANOG, REX1, and FGF4 ${ }^{1}$. Once implanted into the uterine wall, naïve ESCs differentiate towards primed ESCs and continue to express OCT4 and NANOG, alongside FGF5 and $\mathrm{T}^{1}$. Collectively, cells of the epiblast give rise to the embryo proper. As for extraembryonic lineages, the trophectoderm, which gives rise to the placenta, is made up of trophoblast stem cells (TSCs)-expressing $\mathrm{CDX}_{2}{ }^{1}$. The third lineage consist of cells that form extraembryonic endoderm (XEN), which express GATA4, GATA6, SOX7, and $\mathrm{SOX} 17^{2}$. XEN cells differentiate into parietal or visceral endoderm cells, and are essential for the survival and patterning of the embryo ${ }^{2,3}$. Despite the many studies that have focused on the derivation, maintenance, and differentiation of naïve and primed ESCs, fewer have addressed these in regard to extraembryonic lineages, specifically XEN cells.

\section{Mammalian embryos transition through distinct metabolic profiles}

Our understanding of stem cells and their ability to selfrenew and differentiate is corroborated by changes in global gene and protein expression, and the epigenetic modifications. Although these "-omic" approaches provide invaluable insight into the various characteristics that stem cells share, or what makes them unique from other

Correspondence: Gregory M. Kelly (gkelly@uwo.ca)

${ }^{1}$ Molecular Genetics Unit, Department of Biology, The University of Western Ontario, London, ON, Canada

${ }^{2}$ Department of Anatomy and Cell Biology, The University of Western Ontario, London, ON, Canada

Full list of author information is available at the end of the article. cells, one common feature is their pluripotency, which is a topic of ongoing investigation. In the past decade, attention has shifted towards understanding the metabolic landscape of early mammalian embryos ${ }^{4}$. Glucose metabolism provides ATP for energy expenditure and substrates for anabolism that assists in modulating the epigenome. While most somatic cells use mitochondrial oxidative phosphorylation (OXPHOS) to generate ATP, Otto Warburg discovered that, despite having sufficient oxygen levels for OXPHOS metabolism, cancer cells rely on glycolysis to produce $\mathrm{ATP}^{5}$. This phenomenon, termed the Warburg effect, also occurs in stem cells ${ }^{6}$, where naïve ESCs utilize glycolysis and OXPHOS to generate ATP, while primed ESCs are exclusively glycolytic despite having structurally mature mitochondria ${ }^{5}$. Surprisingly, the appearance of these mitochondria in primed ESCs would suggest that they are capable of using OXPHOS, but detailed analysis has revealed that these cells express low levels of cytochrome $c$ oxidase, thus reducing mitochondrial respiratory capacity ${ }^{7}$. As for extraembryonic lineages, we know TSCs use OXPHOS metabolism to generate ATP for energy ${ }^{8}$, and to power the $\mathrm{Na}^{+}$, $\mathrm{K}^{+}$-ATPase pump for blastocoel formation ${ }^{9}$, but we need a better understanding of the metabolic profile(s) in XEN cells to paint a complete picture of the events taking place in early development.

\section{The metabolic state of XEN cells}

Our best understanding of the metabolic landscape of XEN cells comes from proteomic analysis ${ }^{10}$. Rate-limiting enzymes in glycolysis, including hexokinase 2 and glucose transporter 1 , are downregulated during XEN induction; however, other enzymes remain unchanged or are elevated $^{10}$. In fact, we have shown that lactate dehydrogenase A (LDHA), which catalyzes the conversion of pyruvate to lactate, is upregulated in embryo-derived XEN cells, while LDHB, which catalyzes the reverse reaction, is 


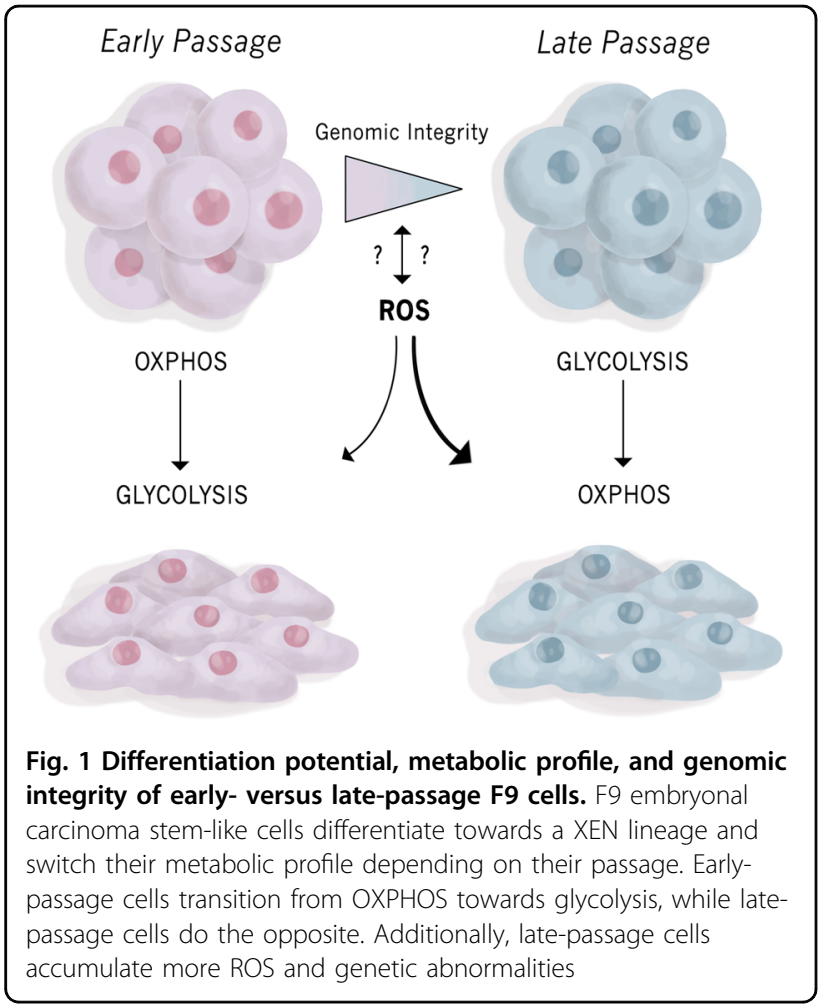

downregulated (unpublished data). Additionally, XEN induction is accompanied by an increase in the levels of enzymes involved in the TCA cycle and electron transport chain (ETC), yet mitochondrial biogenesis proteins are downregulated ${ }^{10}$. These seeming discrepancies suggest that the metabolome of XEN cells might be more complex than that of ESCs and TSCs, and thus further detailed interrogation is warranted.

\section{Factors influencing metabolism, differentiation, and stem cell quality}

F9 embryonal carcinoma stem-like cells differentiate into primitive endoderm when treated with retinoic acid (RA) and to parietal endoderm when treated with RA and dibutyryl cAMP ${ }^{4}$. Our studies and those of others show that this differentiation is accompanied by an increase in GATA6, SOX7, and SOX17, and the decrease of pluripotency genes including, OCT4, REX1, and NANOG ${ }^{4}$. We recently reported that F9 cells differentiate to a XENlike state and this occurs regardless of their passage number ${ }^{11}$. However, it is surprising that the metabolic profile between the early- and late-passage populations differed dramatically. Early-passage cells transitioned from OXPHOS metabolism towards glycolysis, whereas the opposite was seen in late-passage cells. Further examination revealed that there was dysregulation in ETC enzyme stoichiometry in the differentiated late-passage cells, which resulted in the increase in mitochondrial ROS levels. Also, late-passage cells had accumulated chromosomal abnormalities when compared to early-passage cells, and whether changes in the metabolic profile preceded these chromosomal abnormalities or vice versa remains to be determined (Fig. 1) ${ }^{11}$.

\section{Significance and future directions}

Our report in Cell Death Discovery is the first to shed light on the metabolic profile of XEN-like stem cells and how their passaging influences differentiation and metabolism. Mechanistically, the pluripotency potential between the early- versus late-passage populations remains unaltered despite major differences in metabolic states, karyotypes, expression of cell cycle regulators, and proliferation rates. These differences are significant and shed invaluable light as to why it is crucial to determine as many physiological parameters of a stem cell population prior to moving forward its utility as a therapeutic tool for regenerative medicine.

\section{Acknowledgements}

This study was supported by a Discovery Grant (R2615A02) for operating funds from the Natural Sciences and Engineering Research Council of Canada (NSERC) to G.M.K. M.I.G. was supported by NSERC CGS D scholarship.

\section{Author details}

${ }^{1}$ Molecular Genetics Unit, Department of Biology, The University of Western Ontario, London, ON, Canada. ${ }^{2}$ Department of Anatomy and Cell Biology, The University of Western Ontario, London, ON, Canada. ${ }^{3}$ Collaborative Graduate Specialization in Developmental Biology, The University of Western Ontario, London, ON, Canada. ${ }^{4}$ Department of Paediatrics, The University of Western Ontario, London, ON, Canada. ${ }^{5}$ Department of Physiology and Pharmacology, The University of Western Ontario, London, ON, Canada. ${ }^{6}$ Child Health Research Institute, London, ON, Canada. ${ }^{7}$ Ontario Institute for Regenerative Medicine, Toronto, ON, Canada

Conflict of interest

The authors declare that they have no conflict of interest.

\section{Publisher's note}

Springer Nature remains neutral with regard to jurisdictional claims in published maps and institutional affiliations.

Received: 27 September 2018 Accepted: 1 October 2018

Published online: 22 October 2018

\section{References}

1. Kojima, Y., Tam, O. H. \& Tam, P. P. Timing of developmental events in the early mouse embryo. Semin. Cell. Dev. Biol. 34, 65-75 (2014).

2. Plusa, B., Piliszek, A., Frankenberg, S., Artus, J. \& Hadjantonakis, A. K. Distinct sequential cell behaviours direct primitive endoderm formation in the mouse blastocyst. Development 135, 3081-3091 (2008).

3. Rossant, J. \& Tam, P. P. Emerging asymmetry and embryonic patterning in early mouse development. Dev. Cell. 7, 155-164 (2004). 
4. Kelly, G. M. \& Gatie, M. I. Mechanisms regulating stemness and differentiation in embryonal carcinoma cells. Stem Cells Int. 2017, 3684178 (2017).

5. Warburg, O. The metabolism of carcinoma cells. Cancer Res. 9, 148-163 (1925).

6. Spinelli, J. B. \& Haigis, M. C. The multifaceted contributions of mitochondria to cellular metabolism. Nat. Cell Biol. 20, 745-754 (2018).

7. Zhou, W. et al. HIF1a induced switch from bivalent to exclusively glycolytic metabolism during ESC-to-EpiSC/hESC transition. EMBO J. 31, 2103-2116 (2012).
8. Simon, M. C. \& Keith, B. The role of oxygen availability in embryonic development and stem cell function. Nat. Rev. Mol. Cell Biol. 9, 285-296 (2008).

9. Kidder, G. M. \& Watson, A. J. Roles of Na, K-ATPase in early development and trophectoderm differentiation. Semin. Nephrol. 25, 352-355 (2005).

10. Mulvey, C. M. et al. Dynamic proteomic profiling of extra-embryonic endoderm differentiation in mouse embryonic stem cells. Stem Cells 33, 2712-2725 (2015).

11. Gatie, M. I. \& Kelly, G. M. Metabolic profile and differentiation potential of extraembryonic endoderm-like cells. Cell Death Discov. 5, 42-58 (2018). 